\title{
Vidarabine, An Anti-Herpes Agent, Prevents Occlusal-Disharmony-Induced Cardiac Dysfunction in Mice
}

\section{Yoshio Hayakawa}

Tsurumi University School of Dental Medicine

\section{Yoshiki Ohnuki}

Tsurumi University School of Dental Medicine

\section{Kenji Suita}

Tsurumi University School of Dental Medicine

\section{Yasumasa Mototani}

Tsurumi University School of Dental Medicine

\section{Misao Ishikawa}

Tsurumi University School of Dental Medicine

\section{Aiko Ito}

Tsurumi University School of Dental Medicine

\section{Akinaka Morii}

Tsurumi University School of Dental Medicine

\section{Kenichi Kiyomoto}

Tsurumi University School of Dental Medicine Michinori Tsunoda

Tsurumi University School of Dental Medicine Ichiro Matsuo

Tsurumi University School of Dental Medicine Hiroshi Kawahara

Tsurumi University School of Dental Medicine

Satoshi Okumura ( $\nabla$ okumura-s@tsurumi-u.ac.jp )

Tsurumi University School of Dental Medicine

\section{Megumi Nariyama}

Tsurumi University School of Dental Medicine

\section{Research Article}

Keywords: cardiac, vidarabine, occlusal, disharmony, cardiovascular, disease, mice 
Posted Date: July 8th, 2021

DOl: https://doi.org/10.21203/rs.3.rs-646428/v1

License: (c) (1) This work is licensed under a Creative Commons Attribution 4.0 International License. Read Full License

Version of Record: A version of this preprint was published at The Journal of Physiological Sciences on February 11th, 2022. See the published version at https://doi.org/10.1186/s12576-022-00826-4. 


\section{Vidarabine, an anti-herpes agent, prevents}

\section{occlusal-disharmony-induced cardiac dysfunction in mice}

Yoshio Hayakawa ${ }^{1,2}$, Yoshiki Ohnuki ${ }^{1}$, Kenji Suita ${ }^{1}$, Yasumasa Mototani ${ }^{1}$, Misao

Ishikawa $^{3}$, Aiko Ito ${ }^{4}$, Megumi Nariyama ${ }^{5}$, Akinaka Morii ${ }^{1,6}$, Kenichi Kiyomoto ${ }^{1,6}$, Michinori Tsunoda $^{1,6}$, Ichiro Matsuo ${ }^{1,6}$, Hiroshi Kawahara ${ }^{2}$, Satoshi Okumura ${ }^{1}$

${ }^{1}$ Department of Physiology, Tsurumi University School of Dental Medicine, Yokohama 230-8501, Japan

${ }^{2}$ Department of Dental Anesthesiology, Tsurumi University School of Dental Medicine, Yokohama 230-8501, Japan

${ }^{3}$ Department of Oral Anatomy, Tsurumi University School of Dental Medicine, Yokohama 230-8501, Japan

${ }^{4}$ Department of Orthodontics, Tsurumi University School of Dental Medicine, Yokohama 230-8501, Japan

${ }^{5}$ Department of Pediatric Dentistry, Tsurumi University School of Dental Medicine, Yokohama 236-8501, Japan

${ }^{6}$ Department of Periodontology, Tsurumi University School of Dental Medicine, Yokohama 230-8501, Japan 
*Corresponding author: Satoshi Okumura:

Department of Physiology, Tsurumi University School of Dental Medicine,

2-1-3 Tsurumi, Tsurumi-ku, Yokohama 230-8501; (Tel. +81-(0)45-580-8476;

Fax. +81-(0)45-585-2889; e-mail: okumura-s@ @surumi-u.ac.jp)

Word count: 7,540

7 figures, 1 table and 13 supplementary figures 


\section{Abstract}

We recently reported a positive relationship between occlusal disharmony and cardiovascular disease via activation of $\beta$-adrenergic signaling in mice. Furthermore, inhibition of type 5 adenylyl cyclase (AC5), a major cardiac subtype in adults, protects the heart against oxidative stress. Here, we examined the role of AC5 in the development of occlusal-disharmony-induced cardiovascular disease in bite-opening (BO) mice, prepared by cementing a suitable appliance onto the mandibular incisor. We first examined the effects of BO treatment on cardiac function in mice treated or not treated for 2 weeks with vidarabine, which we previously identified as an inhibitor of cardiac AC. Cardiac function was significantly decreased in the BO group compared to the control group, but vidarabine ameliorated the dysfunction. Cardiac fibrosis, myocyte apoptosis and myocyte oxidative DNA damage were significantly increased in the BO group, but vidarabine blocked these changes. The BO-induced cardiac dysfunction was associated with increased phospholamban phosphorylation at threonine-17 and serine-16, as well as increased activation of the $\mathrm{Ca}^{2+}$-calmodulin-dependent protein kinase II/receptor-interacting protein 3 signaling pathway. These data suggest that AC5 inhibition with vidarabine might be a new therapeutic approach for the treatment of cardiovascular disease associated with occlusal disharmony. 


\section{Introduction}

Oral health decrease with age, and this is a major risk factor for many diseases, including cardiovascular disease ${ }^{1}$. In addition, aging-related loss of teeth, poor periodontal status and low alveolar bone levels can lead to occlusal disharmony ${ }^{1,2}$. Increased sympathetic nervous activity was recently recognized as a hallmark feature that links aging with increased cardiovascular risk ${ }^{3}$, and it is also involved in the etiology of oral frailty ${ }^{4-6}$.

Adenylyl cyclase (AC) is the target enzyme of $\beta$-adrenergic receptor ( $\beta$-AR) signaling stimulation. At least 9 isoforms are known, 7 of which are expressed in the heart; the type 5 isoform (AC5) is a major adult cardiac isoform, while type 6 is a major fetal isoform ${ }^{7-9}$. We have developed a mouse model with knock-out of type 5 adenylyl cyclase $(\mathrm{AC} 5 \mathrm{KO}){ }^{10}$ and we have also identified vidarabine as a cardiac AC inhibitor in mice ${ }^{11}$. Using these models, we found that genetic disruption and pharmacological inhibition of AC5 are associated with resistance to the development of heart failure $10,12,13$ and increased longevity ${ }^{13,14}$.

We previously examined the effects of occlusal disharmony on cardiac remodeling (fibrosis and myocyte apoptosis), cardiac function and susceptibility to atrial fibrillation in bite-opening mice (BO), in which a $0.7 \mathrm{~mm}$ BO was introduced by cementing a 
suitable appliance onto the mandibular incisor. We found that BO-induced cardiac dysfunction and susceptibility to atrial fibrillation are ameliorated by co-treatment with propranolol, a non-selective $\beta$-blocker ${ }^{15,16}$. These findings indicate that activation of $\beta$-AR signaling might play a part in the cardiac dysfunction induced by occlusal disharmony.

However, the role of AC5 in the occlusal-disharmony-induced cardiac deterioration remains poorly understood. In this study, therefore, we examined the effects of AC5 inhibition with vidarabine on cardiac function, cardiac fibrosis, myocyte apoptosis, and oxidative DNA damage induced by occlusal disharmony in mice (Fig. 1A, B, Supplementary Fig. 1). Acute and chronic treatment with vidarabine does not alter basal cardiac function in healthy animals, unlike $\beta$-blocker administration ${ }^{17,18}$. More importantly, vidarabine has been used as an anti-viral drug for many years in humans 11,17. Therefore, vidarabine, rather than $\beta$-blocker, might be a safe and immediately clinically available drug for the treatment or prevention of cardiac dysfunction induced by occlusal disharmony. 


\section{Results}

\section{Effects of BO on body weight with/without vidarabine co-treatment.}

There was no significant difference of body weight (BW) between the control and vidarabine groups during the experimental period (Fig. 1C). However, postoperative $\mathrm{BW}$ initially decreased in the $\mathrm{BO}$ and $\mathrm{BO}$ plus vidarabine treatment groups $(\mathrm{BO}+$ vidarabine), reaching a minimum at 4-6 days after the BO treatment, in accordance with

previous findings by our group ${ }^{15}$ and others ${ }^{19,20}$. Then, the postoperative BW of the BO and $\mathrm{BO}+$ vidarabine groups gradually increased, but did not recover to the preoperative level within the experimental period of 2weeks (Fig. 1C).

\section{Effects of BO on heart size and lung weight with/without vidarabine co-treatment.}

We examined the effect of $\mathrm{BO}$ with/without vidarabine on heart size in terms of cardiac muscle mass per tibial length ratio (Fig. 1D) and the effect on wet lung weight in terms of wet lung weight per tibial length ratio (Fig. 1E). Similar results were obtained among the four groups $(n=5$ each).

These data suggest that BO did not induce cardiac hypertrophy or lung edema during the experimental period. 


\section{Vidarabine inhibits BO-induced cardiac fibrosis.}

We examined cardiac fibrosis in $\mathrm{BO}$ mice with/without vidarabine by means of Masson-trichrome staining (Fig. 2A and B). BO treatment significantly increased the area of fibrosis in cardiac muscle (Control $(n=4)$ vs. BO $(n=5) ; 0.9 \pm 0.4 \%$ vs. $2.5 \pm$ $0.8 \%, P<0.01$ vs. Control) in accordance with our previous finding ${ }^{15}$. Vidarabine alone did not alter the area of fibrosis, but it blocked the BO-induced increase of fibrosis $(\mathrm{BO}(n=5)$ vs. $\mathrm{BO}+$ vidarabine $(n=4) ; 2.5 \pm 0.8 \%$ vs. $1.1 \pm 0.5 \%, P<0.001$ vs. BO).

We also evaluated cardiac fibrosis by measuring the level of $\alpha$-smooth muscle actin ( $\alpha$-SMA) expression at 2 weeks after the start of BO, because this parameter is closely associated with cardiac fibrosis ${ }^{21}$. Expression of $\alpha$-SMA was significantly increased in cardiac muscle of BO mice (Control $(n=5)$ vs. BO $(n=4) ; 1.0 \pm 0.4$ vs. $2.5 \pm 0.9$ A.U., $P<0.05$ vs. Control), and the increase was significantly suppressed by vidarabine (BO $(n=4)$ vs. $\mathrm{BO}+$ vidarabine $(n=4) ; 2.5 \pm 0.9$ vs. $0.7 \pm 0.5$ A.U., $P<0.01$ vs. BO) (Fig. 2C).

These data, together with our previous findings ${ }^{17}$, suggest that cardiac fibrosis induced by BO might be mediated at least in part through the activation of AC5. 


\section{Vidarabine inhibits BO-induced cardiac myocyte apoptosis.}

We also examined cardiac myocyte apoptosis in BO mice with/without vidarabine by means of terminal deoxyribonucleotidyl transferase (TdT)-mediated biotin-16-deoxyuridine (TUNEL) staining (Fig. 3A and B). BO treatment significantly increased cardiac myocyte apoptosis (Control $(n=4)$ vs. BO $(n=5) ; 1.6 \pm 1.3 \%$ vs. 4.2 $\pm 1.7 \%, P<0.05$ vs. Control) in accordance with our previous findings ${ }^{15}$. Vidarabine alone $(n=5)$ had no effects on the number of TUNEL-positive cardiac myocytes, but it blocked the BO-induced increase of TUNEL-positive cardiac myocytes (BO $(n=5)$ vs. $\mathrm{BO}+$ vidarabine $(n=4) ; 4.2 \pm 1.7 \%$ vs. $1.3 \pm 0.7 \%, P<0.05$ vs. Control $)$.

We also evaluated cardiac myocyte apoptosis by examining the ratio of BCL-2 associated X (Bax) protein, an accelerator of apoptosis, and B cell lymphoma 2 (BCL-2 ) protein, a regulator of apoptosis, in the heart (Fig. 3C).

BO treatment significantly increased the ratio of Bax/BCL-2 in the heart (Control $(n=4)$ vs. $\mathrm{BO}(n=4) ; 1.0 \pm 0.6 \%$ vs. $5.9 \pm 2.6$ A.U., $P<0.05$ vs. Control). Vidarabine alone $(n=5)$ had no effects on the ratio of Bax/BCL-2, but it blocked the BO-induced increase in the $\mathrm{Bax} / \mathrm{BCL}-2$ ratio $(\mathrm{BO}(n=4)$ vs. $\mathrm{BO}+$ vidarabine $(n=5) ; 5.9 \pm 2.6$ vs. $1.3 \pm 1.0$ A.U., $P<0.05$ vs. Control) (Fig. 3C).

These and earlier results ${ }^{11}$ support the idea that the increase of TUNEL-positive 
cardiac myocytes induced by $\mathrm{BO}$ treatment might be mediated at least in part through the activation of AC5.

\section{Vidarabine inhibits BO-induced cardiac dysfunction.}

We performed echocardiography to evaluate cardiac function in terms of left ventricular cardiac function (EF) and fractional shortening (\%FS) (Table 1). Both parameters were significantly decreased in the $\mathrm{BO}$ group compared to the control mice (EF: Control $(n=6)$ vs. BO $(n=6) ; 68 \pm 1.1 \%$ vs. $61 \pm 3.0 \%, P<0.001$ vs. Control; \%FS: Control $(n=6)$ vs. $\mathrm{BO}(n=6) ; 33 \pm 0.8 \%$ vs. $28 \pm 1.9 \%, P<0.001$ vs. Control). Vidarabine alone $(n=7)$ had no effect on EF or $\% \mathrm{FS}$, but blocked the BO-induced decrease of EF and \%FS (EF: BO $(n=6)$ vs. BO + vidarabine $(n=6) ; 61 \pm$ $3.0 \%$ vs. $66 \pm 1.4 \%, P<0.01$ vs. Control; $\%$ FS: $\mathrm{BO}(n=6)$ vs. $\mathrm{BO}+$ vidarabine $(n=$ 6); $28 \pm 1.9 \%$ vs. $31 \pm 1.0 \%, P<0.01$ vs. Control).

These data suggest that BO treatment decreases cardiac function at least in part through the activation of AC5.

Vidarabine inhibits BO-induced oxidative stress.

We examined oxidative stress in the myocardium by 
8-hydroxy-2'-deoxyguanosine (8-OHdG) immunostaining (Fig. 4A and B) and western blotting of oxidized proteins (Fig. 4C and D).

We first prepared positive and negative control cross sections by incubation with (positive control)/without (negative control) $0.3 \% \mathrm{H}_{2} \mathrm{O}_{2}$ in TBS-T for $1 \mathrm{~h}$ at room temperature before the step of anti-8-OHdG antibody treatment. The 8-OHdG staining procedure could clearly discriminate $8-\mathrm{OHdG}$-positive and non-positive nuclei, thereby confirming the validity (Supplementary Fig. 4).

The ratio of 8-OHdG-positive/total cells was significantly increased in the BO group (Control $(n=4)$ vs. $\mathrm{BO}(n=5) ; 1.1 \pm 0.6 \%$ vs. $3.0 \pm 1.4 \%, P<0.05$ vs. Control), and the increase was suppressed by vidarabine (BO $(n=5)$ vs. $\mathrm{BO}+$ vidarabine $(n=4)$; $3.0 \pm 1.4 \%$ vs. $1.1 \pm 0.4 \%, P<0.05$ vs. Control).

We also measured the amount of oxidized proteins using the OxiSelect TM protein kit and confirmed that there was a significant increase (Control $(n=4)$ vs. BO $(n=5)$; $1.0 \pm 0.1$ vs. $2.7 \pm 1.0$ A.U., $P<0.01$ vs. Control). However, the increase was again suppressed by vidarabine (BO $(n=5)$ vs. $\mathrm{BO}+$ vidarabine $(n=4) ; 2.7 \pm 1.0$ vs. $1.1 \pm$ 0.4 A.U., $P<0.05$ vs. Control).

These results, together with the data shown in Fig. 2, Fig. 3 and Table 1, indicate that BO treatment increases oxidative stress-induced myocardial damage at least in part 
through the activation of AC5, which might contribute to the cardiac remodeling and dysfunction in $\mathrm{BO}$ mice.

\section{Vidarabine inhibits BO-mediated NOX4 expression.}

Reactive oxygen species (ROS) are produced through a number of pathways, including nicotinamide adenine dinucleotide phosphate oxidase (NOX) and xanthine oxidase (XO), and may be involved in various physiological and pathological processes in the heart, including fibrosis, apoptosis and heart failure ${ }^{22-25}$.

Two NOX isoforms, NOX2 and NOX4, are expressed in the heart, and their activity is regulated by their expression level ${ }^{23,26}$. We therefore examined NOX4 and NOX2 protein expression in the heart among the four groups. NOX4 expression was significantly increased in the BO group (Control $(n=4)$ vs. BO $(n=4) ; 1.0 \pm 0.3$ vs. 2.0 \pm 0.2 A.U., $P<0.001$ vs. Control). This increase was significantly inhibited by vidarabine $(\mathrm{BO}(n=4)$ vs. $\mathrm{BO}+$ vidarabine $(n=5) ; 2.0 \pm 0.2$ vs. $1.3 \pm 1.0$ A.U., $P<$ 0.01 vs. Control) (Fig. 5A). We also examined NOX2 protein expression and found that it was similar among the four groups $(n=5$ each) (Fig. 5B).

We next examined XO expression in the heart in the four groups. XO expression was significantly increased in the BO group (Control $(n=4)$ vs. BO $(n=4) ; 1.0 \pm 0.3$ vs. 
$2.5 \pm 0.8$ A.U., $P<0.01$ vs. Control). This increase was significantly inhibited by vidarabine $(\mathrm{BO}(n=4)$ vs. $\mathrm{BO}+$ vidarabine $(n=4) ; 2.5 \pm 0.8$ vs. $0.9 \pm 0.4$ A.U., $P<$ 0.001 vs. Control) (Fig. 5C).

These data suggested that NOX4 and XO might contribute to the activation of AC5 and thus to the development of BO-mediated oxidative stress and pathological remodeling in the heart

\section{Vidarabine inhibits BO-mediated activation of p38 MAPK.}

Overproduction of ROS derived from NOX4 and XO triggers oxidative stress and subsequently activates the p38 mitogen-activated protein kinase (MAPK) signaling pathway, leading to cardiac dysfunction ${ }^{26-28}$. We therefore examined the phosphorylation levels of p38 MAPK and found that p38 MAPK phosphorylation was significantly increased in the BO group (Control $(n=4)$ vs. $\mathrm{BO}(n=5) ; 1.0 \pm 0.3$ vs. 2.8 \pm 1.7 A.U., $P<0.05$ vs. Control). This increase was significantly inhibited by vidarabine $(\mathrm{BO}(n=5)$ vs. $\mathrm{BO}+$ vidarabine $(n=5) ; 2.8 \pm 1.7$ vs. $1.1 \pm 0.4$ A.U., $P<$ 0.05 vs. Control) (Fig. 5D).

These data suggest that overproduction of ROS derived from BO-induced upregulation of NOX4 and XO activates the p38 MAPK signaling pathway, which 
might lead to cardiac remodeling and dysfunction.

\section{Vidarabine inhibits BO-induced necroptosis.}

Overproduction of ROS derived from NOX4 was recently demonstrated to cause myocardial apoptosis and necroptosis, leading to heart failure via activation of receptor-interacting protein 3 (RIP3) in cardiac myocytes ${ }^{29}$. We therefore examined the amount of phospho-RIP3 (Thr-231/Ser-232) and found that it was significantly increased in the heart of BO mice (Control $(n=5)$ vs. $\mathrm{BO}(n=4) ; 1.0 \pm 0.3$ vs. $5.4 \pm 2.3$ A.U., $P<0.001$ vs. Control) (Fig. 6A). Again, this increase was significantly attenuated by vidarabine $(\mathrm{BO}(n=4)$ vs. $\mathrm{BO}+$ vidarabine $(n=4) ; 5.4 \pm 2.3$ vs. $1.7 \pm 0.5$ A.U., $P<$ 0.01 vs. Control) (Fig. 6A).

These data suggest that BO treatment might increase RIP3 phosphorylation, leading to apoptosis and necroptosis through the activation of AC5 in the heart.

\section{Vidarabibe inhibits BO-induced CaMKII phosphorylation.}

RIP3-mediated myocardial apoptosis and necroptosis were recently demonstrated to be dependent upon the activation of calmodulin kinase II (CaMKII) ${ }^{29}$. We therefore examined the amount of phospho-CaMKII (Thr-286) (Fig. 6B) in the heart of BO mice 
and found that it was significantly increased at two weeks after BO (Control $(n=4)$ vs. BO $(n=5) ; 1.0 \pm 0.2$ vs. $1.9 \pm 0.2$ A.U., $P<0.001$ vs. Control $)$. The increase was significantly attenuated by vidarabine (BO $(n=5)$ vs. BO + vidarabine $(n=4) ; 1.9 \pm 0.2$ vs. $1.2 \pm 0.3, P<0.01$ vs. Control).

These data suggest that $\mathrm{BO}$ treatment might activate NOX4/RIP3/CaMKII signaling via activation of AC5.

\section{Vidarabine inhibits BO-induced PLN phosphorylation.}

The elevation of diastolic sarcoplasmic reticulumn $\mathrm{Ca}^{2+}$ leakage mediated by phospholamban (PLN) phosphorylation contributes considerably to the pathogenesis of cardiac remodeling and dysfunction via ROS derived from NOX4 ${ }^{30,31}$. We therefore examined the effect of BO on PLN phosphorylation at Thr-17 and Ser-16, which are phosphorylated by CaMKII and by protein kinase A (Fig. 6C and D).

Phospho-PLN (Thr-17) was significantly increased in cardiac muscle of BO mice (Control $(n=4)$ vs. BO $(n=5) ; 1.0 \pm 0.2$ vs. $2.6 \pm 1.3$ A.U., $P<0.05$ vs. Control) (Fig. 6C). This increase was significantly attenuated by vidarabine $(\mathrm{BO}(n=5)$ vs. $\mathrm{BO}+$ vidarabine $(n=5) ; 2.6 \pm 1.3 \%$ vs. $0.7 \pm 0.2$ A.U., $P<0.01$ vs. BO) (Fig. 6C).

Phospho-PLN (Ser-16) was also significantly increased in cardiac muscle of BO 
mice (Control $(n=4)$ vs. BO $(n=5) ; 1.0 \pm 0.5$ vs. $2.2 \pm 0.8$ A.U., $P<0.05$ vs. Control). Again, the increase was significantly attenuated by vidarabine (BO $(n=5)$ vs. $\mathrm{BO}+$ vidarabine ( $n=5) ; 2.2 \pm 0.8$ vs. $0.8 \pm 0.1$ A.U., $P<0.01$ vs. $\mathrm{BO})$.

These data, together with the previous results, suggest that BO might increase PLN phosphorylation, leading to ROS-mediated elevation of diastolic sarcoplasmic reticulum $\mathrm{Ca}^{2+}$ leakage in cardiac myocytes. 


\section{Discussion}

Oral health is important for maintaining general health and is associated with physical activity, including the status of the cardiovascular system ${ }^{32}$. Extensive studies have shown that the link between oral health and cardiovascular disease (CVD) may be explained by chronic inflammation and repeated bacteremia from the oral cavity, as inflammation plays an important role in the pathogenesis of CVD ${ }^{1}$. However, periodontal status may not completely explain the oral health-CVD relationship, and other factors such as occlusal disharmony, might also contribute ${ }^{33}$.

Occlusal disharmony due to BO treatment causes muscle dysfunction and susceptibility to muscle fatigue in masseter and suprahyoid muscles via accumulation of ROS in rats ${ }^{34,35}$. In addition, occlusal-disharmony-induced ROS production in the oral cavity might cause not only local pathogenic disturbance, but also systemic diseases, including heart disease, in patients ${ }^{36}$. Importantly, we previously showed that cardiac fibrosis and myocyte apoptosis were significantly increased in cardiac muscle of BO mice, together with accumulation of ROS, leading to cardiac dysfunction and susceptibility to atrial fibrillation via activation of $\beta$-AR signaling ${ }^{15,16}$. Here, we examined whether AC5, a major cardiac AC isoform, is a mediator of the $\beta$-AR signaling leading to occlusal-disharmony-induced cardiac dysfunction, because AC5 is 
known to be a major regulator of oxidative stress in the heart ${ }^{37,38}$.

AC transduces the signal generated by binding of a ligand, most commonly norepinephrine, to $\beta$-AR and Gs $\alpha$, resulting in the conversion of ATP to cAMP ${ }^{39}$. There are nine major mammalian isoforms of AC, with AC6 being the major fetal cardiac AC isoform, and AC5, the major cardiac isoform in adults ${ }^{9,39,40}$. We showed that in type 5 AC knockout mice (AC5KO), the heart was protected against the stress of chronic pressure overload ${ }^{41}$ and chronic catecholamine stimulation ${ }^{12}$. Interestingly, however, AC5 inhibition appears to have multiple effects. For example, AC5 gene knockout or pharmacological AC5 inhibition increases longevity in mice ${ }^{14,17}$. Inhibition of AC5 also protects against physical frailty, enhances exercise capacity, and protects against diabetes, obesity and diabetic cardiomyopathy ${ }^{14,17,37}$.

Several epidemiological surveys have confirmed a positive relationship between oral health and physical frailty, including cardiovascular disease ${ }^{1,42}$. Tooth loss could contribute to occlusal disharmony or impaired masticatory performance ${ }^{43-45}$. More recently, a relationship between occlusal disharmony and cardiovascular disease was demonstrated in a Japanese urban population ${ }^{1,46}$. However, the mechanism remains poorly understood.

In this study, pharmacological AC5 inhibition with vidarabine was shown to 
protect the heart from occlusal-disharmony-induced oxidative stress in BO mice, a well-established model of occlusal disharmony ${ }^{15,16,19,47}$. In particular, our findings indicate that AC5 inhibition in BO mice has a cardioprotective effect mediated at least in part by a decrease of NOX4 overexpression and CaMKII phosphorylation, leading to phospholamban phosphorylation on serine 16 and threonine 17 (Fig. 7).

We previously identified vidarabine as a candidate AC5 inhibitor by computer-based drug screening, and its inhibitory activity was confirmed in an in vitro cAMP accumulation assay in cardiac myocytes and also in AC assay using a crude membrane preparation ${ }^{11,17,48}$. The major finding of the current investigation is that inhibition of AC5 by vidarabine ameliorates the development of occlusal-disharmony-induced cardiac dysfunction by reducing the oxidative stress generated via the RIP3/NOX4 signaling pathway in a cAMP-dependent mechanism. We have previously reported that vidarabine inhibits the development of catecholamine-induced heart failure and atrial fibrillation in mice without suppressing cardiac function ${ }^{17,49}$. Our current and previous findings suggest that vidarabine might broadly inhibit stress-induced cardiomyopathy, leading to improved longevity and reduced physical frailty. Since vidarabine is a clinically approved drug, an early clinically trial should be feasible. 


\section{Materials and methods}

\section{Mice and experimental protocols}

All experiments were performed on male 12-week-old C57BL/6 mice obtained from CLEA Japan (Tokyo, Japan). Mice were group-housed at $23^{\circ} \mathrm{C}$ under a $12-12$ light/dark cycle with lights on at 8:00 AM in accordance with the standard conditions for mouse studies by our group ${ }^{15,16,50,51}$. Both food and water were available ad libitum.

Occlusal disharmony in mice was induced by introducing a 0.7-mm BO,

employing our standard method of cementing a suitable appliance onto the mandibular incisor under intraperitoneal anesthesia with medetomidine $(0.03 \mathrm{mg} / \mathrm{ml})$, midazolam $(0.4 \mathrm{mg} / \mathrm{ml})$, and butorphanol $(0.5 \mathrm{mg} / \mathrm{ml}){ }^{15,16,19}$. Mice were divided into four groups: a normal control group (Control), a BO-only treatment group (BO), a vidarabine-only treatment group $(\mathrm{V})$, and a $\mathrm{BO}$ plus vidarabine treatment group $(\mathrm{BO}+\mathrm{V})($ Fig. $\mathbf{1 A}$ and B). Chronic infusion of vidarabine dissolved in DMSO (\#359-13471; Sigma, St. Louis MO, USA) was performed for 14 days at a dose of $15 \mathrm{mg} / \mathrm{kg} /$ day delivered with osmotic mini-pumps (Model 2002; ALZET, Cupertino, CA, USA) ${ }^{17,52}$. The dose of vidarabine was selected based upon that used in previous studies ${ }^{17,52}$. Because the BO mice cannot easily eat the standard pellet food (CE-2: $334.9 \mathrm{kcal} / 100 \mathrm{~g}$; CLEA Japan), but can take paste food, the standard pellet food was changed to paste food three days before the BO 
treatment in all groups, as in previous studies ${ }^{19,53}$. BW was monitored throughout the 2-week experimental period ( $n=5$ each) (Fig. 1C).

\section{Ethical approval}

All animal experiments complied with the ARRIVE guidelines ${ }^{54}$ and were carried out in accordance with the National Institutes of Health guide for the care and use of laboratory animals ${ }^{55}$ and institutional guidelines. The experimental protocol was approved by the Animal Care and Use Committee of Tsurumi University (No. 29A041).

\section{Physiological experiments}

Mice (Control: $n=6$, BO: $n=6, \mathrm{~V}: n=7, \mathrm{BO}+\mathrm{V}: n=6$ ) were anesthetized via a mask with isoflurane vapor $(1.0-1.5 \% \mathrm{v} / \mathrm{v})$ titrated to maintain the lightest anesthesia possible, and echocardiographic measurements were performed by means of ultrasonography (TUS-A300, Toshiba, Tokyo, Japan) at 14 days after BO treatment ${ }^{56}$.

\section{Evaluation of fibrosis}

Among several quantitative methods available to determine interstitial fibrotic regions ${ }^{51,57,58}$, we employed Masson-trichrome staining using the Accustatin Trichrome 
Stain Kit (\#HT15-1KT; Sigma-Aldrich, St. Louis, MO, USA) in accordance with the manufacturer's protocol, as described previously ${ }^{15,16,50,51}$. Cross sections $(10 \mu \mathrm{m})$ were cut with a cryostat (CM1900; Leica Microsystems, Nussloch, Germony). The sections were air-dried and fixed with $4 \%$ paraformaldehyde (v/v) in $0.1 \mathrm{M}$ phosphate-buffered saline (pH 7.5) (Control: $n=4$, BO: $n=5, \mathrm{~V}: n=4, \mathrm{BO}+\mathrm{V}: n=4)^{15,16,50,51}$. We quantified interstitial fibrotic regions using freely available image analysis software (Image J 1.48; https://imagej.nih.gov/ij/download.html) to evaluate the percentage of blue area in the Masson-trichrome sections ${ }^{15,16,50,51}$.

\section{Evaluation of apoptosis}

Apoptosis was determined by TUNEL staining using an Apoptosis in situ Detection Kit (\#293-71501; Wako, Osaka, Japan). TUNEL-positive nuclei per field of view were manually counted in six sections of each of the four groups (Control: $n=4$, BO: $n=5, \mathrm{~V}: n=5, \mathrm{BO}+\mathrm{V}: n=4)$ over a microscopic field of $20 \mathrm{x}$, then averaged and expressed as the ratio of TUNEL-positive nuclei $(\%){ }^{41,56}$. By limiting the counting of total nuclei and TUNEL-positive nuclei to areas containing true cross sections of myocytes, we could selectively count only those nuclei that were clearly located within myocytes. 


\section{Western blotting}

Cardiac muscle excised from mice (Control: $n=5$, BO: $n=5$, V: $n=5$, BO $+\mathrm{V}: n$ = 5) was homogenized in a Polytron (Kinematica AG, Lucerne, Switzerland) in ice-cold RIPA buffer (Thermo Fisher Scientific, Waltham, MA, USA: 25 mM Tris-HCl (pH 7.6), $150 \mathrm{mM} \mathrm{NaCl}, 1 \% \mathrm{NP}-40,1 \%$ sodium deoxycholate, $0.1 \%$ SDS) without addition of inhibitors ${ }^{59}$, and the homogenate was centrifuged at $13,000 \mathrm{x} g$ for $10 \mathrm{~min}$ at $4^{\circ} \mathrm{C}$. The supernatant was collected and the protein concentration was measured using a DC protein assay kit (Bio-Rad, Hercules, CA, USA). Equal amounts of protein $(5 \mu \mathrm{g})$ were subjected to $12.5 \%$ SDS-polyacrylamide gel electrophoresis and blotted onto $0.2 \mathrm{~mm}$ PVDF membrane (Millipore, Billerica, MA, USA).

Western blotting was conducted with commercially available antibodies ${ }^{10,41,56}$. Primary antibodies against $\alpha$-SMA (1:1000, \#19245), CaMKII (1:1000, \#3362), phospho-CaMKII (1:1000, Thr-286, \#3361), BCL-2 (1:1000, \#3498), BAX (1:1000, \#2772), RIP3 (1:1000, \#95702), phospho-RIP3 (1:1000, Thr-231/Ser-232, \#91702), p38 (1:1000, \#8690) and phospho-p38 (1:1000, Thr-180/Tyr-182, \#4511) were purchased from Cell Signaling Technology (Boston, MA, USA), primary antibodies against glyceraldehyde-3-phosphate dehydrogenase (GAPDH) (1:200, sc-25778) were 
purchased from Santa Cruz Biotechnology (Santa Cruz, CA, USA) and primary

antibodies against phospho-PLN (1:5000, Thr-17, \#A010-13; 1:5000, Ser-16,

\#A010-12) and PLN (1:5000, \#A010-14) were purchased from Badrilla (Leeds, UK).

Primary antibodies against NOX4 (1:1000, \#ab133303), NOX2 (1:1000, \#ab80508) and

XO (1:1000, \#ab109235) were purchased from Abcam (Cambridge, UK). Horseradish

peroxide-conjugated anti-rabbit (1:5000, \#NA934) or anti-mouse IgG (1:5000,

\#NA931) purchased from GB Healthcare was used as the secondary antibody. The

primary and secondary antibodies were diluted in Tris-buffered saline ( $\mathrm{pH} 7.6)$ with

$0.1 \%$ Tween 20 and $5 \%$ bovine serum albumin. The blots were visualized with

enhanced chemiluminescence solution (ECL: Prime Western Blotting Detection

Reagent, GE Healthcare, Piscataway, NJ, USA) and scanned with a densitometer

(LAS-1000, Fuji Photo Film, Tokyo, Japan). Note that there are different numbers of samples in different western blotting figures (Figs. 2-6) because we excluded outliers (extremely low or high values compared to others in the same groups).

\section{Immunostaining}

Oxidative DNA damage in the myocardium was evaluated by immunostaining for 8-OHdG using the Vector M.O.M Immunodetection system (Control: $n=4$, BO: $n=5$, 
$\mathrm{V}: n=5, \mathrm{BO}+\mathrm{V}: n=4)(\# \mathrm{PK}-2200$, Vector Laboratories, Inc. Burlingame, CA, USA) under our standard conditions ${ }^{15,16}$. Cross sections were cut at $10 \mu \mathrm{m}$ with a cryostat at $-20^{\circ} \mathrm{C}$, air-dried and fixed with $4 \%$ paraformaldehyde (v/v) in TBS-T for $5 \mathrm{~min}$ at room temperature. Antigen retrieval was achieved with $0.1 \%$ citrate plus $1 \%$ Triton $\mathrm{X}-100$ for $30 \mathrm{~min}$ at room temperature, then the sections were washed with TBS-T, incubated with $0.3 \%$ horse serum in TBS-T for $1 \mathrm{~h}$ at room temperature, and blocked with M.O.M. blocking reagent (Vector Laboratories, Burlingame, CA, USA) overnight at $4^{\circ} \mathrm{C}$. For the positive control, sections were incubated with $0.3 \% \mathrm{H}_{2} \mathrm{O}_{2}$ in TBS-T before the anti-8-OHdG antibody treatment. The sections were incubated with anti-8-OHdG antibody $(8.3 \mu \mathrm{g} / \mathrm{ml}$ in M.O.M. Dilute; clone N45.1 monoclonal antibody; Japan Institute for the Control of Aging, Shizuoka, Japan) overnight at $4^{\circ} \mathrm{C}$ in a humidified chamber, then incubated with $0.3 \% \mathrm{H}_{2} \mathrm{O}_{2}$ in $0.3 \%$ horse serum for $1 \mathrm{~h}$ at room temperature to inactivate endogenous peroxidase, rinsed with TBS-T, incubated with anti-mouse IgG in M.O.M. Diluent, and processed with an ABC kit (Vector Laboratories, Inc. Burlingame, CA, USA). The ratio of 8-OHdG nuclei with oxidative DNA damage (stained dark brown) per total cell number was evaluated.

\section{Statistical analysis}


Data are presented as means \pm standard deviation (SD). Comparisons were performed using two-way repeated-measures ANOVA followed by the Bonferroni post hoc test (Fig. 1 C) or one-way ANOVA followed by Tukey's post hoc test (Fig. 1D, 1E, 2B, 2C, 3B, 3C, 4B, 4D, 5A-5D, 6A-6D). Differences were considered significant when $P<0.05$. 


\section{Competing interests}

The authors declare that they have no conflict of interest.

\section{Acknowledgements}

This study was supported in part by the Japan Society for the Promotion of Science (JSPS) KAKENHI Grant (20K10304 to YO, 20K10305 to KS, 19K24109, 21K17171 to $\mathrm{AI}$ and $18 \mathrm{~K} 06862,21 \mathrm{~K} 10242$ to SO)

\section{Author contributions}

Y.H., Y.O., K.S., S.O. conceived and designed the research. Y.H., K.S., M.I., A.I., I.M. performed the experiments. Y.M., M.N., A.M., K.K., M.T., H.K. contributed reagents/materials/analysis tools. S.O. wrote the paper. All authors have read and approved the final manuscript. 


\section{References}

1 Ogawa, M. et al. Relationship between oral health and physical frailty in patients with cardiovascular disease. J Cardiol 77, 131-138, doi:10.1016/j.jjcc.2020.07.016 (2021).

2 An, J. Y., Darveau, R. \& Kaeberlein, M. Oral health in geroscience: animal models and the aging oral cavity. Geroscience 40, 1-10, doi:10.1007/s11357-017-0004-9 (2018).

3 Balasubramanian, P., Hall, D. \& Subramanian, M. Sympathetic nervous system as a target for aging and obesity-related cardiovascular diseases. Geroscience 41, 13-24, doi:10.1007/s11357-018-0048-5 (2019).

4 Segovia, M., Ganzinelli, S., Reina, S., Borda, E. \& Sterin-Borda, L. Role of anti- $\beta_{1}$ adrenergic antibodies from patients with periodontitis in cardiac dysfunction. J. Oral Pathol. Med. 41, 242-248, doi:10.1111/j.1600-0714.2011.01090.x (2012).

5 Collin, H. L. et al. Oral symptoms and signs in elderly patients with type 2 diabetes mellitus. A focus on diabetic neuropathy. Oral Surg. Oral. Med. Oral Pathol. Oral Radiol. Endod. 90, 299-305, doi:10.1067/moe.2000.107536 (2000).

6 Shimizu, Y., Hosomichi, J., Kaneko, S., Shibutani, N. \& Ono, T. Effect of 
sympathetic nervous activity on alveolar bone loss induced by occlusal hypofunction in rats. Arch. Oral Biol. 56, 1404-1411, doi:10.1016/j.archoralbio.2011.05.004 (2011).

7 Ishikawa, Y. et al. Isolation and characterization of a novel cardiac adenylylcyclase cDNA. J. Biol. Chem. 267, 13553-13557 (1992).

8 Katsushika, S. et al. Cloning and characterization of a sixth adenylyl cyclase isoform: types V and VI constitute a subgroup within the mammalian adenylyl cyclase family. Proc. Natl. Acad. Sci. USA 89, 8774-8778, doi:10.1073/pnas.89.18.8774 (1992).

9 Tobise, K. et al. Changes in type VI adenylyl cyclase isoform expression correlate with a decreased capacity for cAMP generation in the aging ventricle. Circ. Res. 74, 596-603, doi:10.1161/01.res.74.4.596 (1994).

10 Okumura, S. et al. Type 5 adenylyl cyclase disruption alters not only sympathetic but also parasympathetic and calcium-mediated cardiac regulation. Circ. Res. 93, 364-371, doi:10.1161/01.res.0000086986.35568.63 (2003).

11 Iwatsubo, K. et al. Direct inhibition of type 5 adenylyl cyclase prevents myocardial apoptosis without functional deterioration. J. Biol. Chem. 279, 40938-40945, doi:10.1074/jbc.M314238200 (2004). 
12 Okumura, S. et al. Disruption of type 5 adenylyl cyclase enhances

desensitization of cyclic adenosine monophosphate signal and increases Akt signal with chronic catecholamine stress. Circulation 116, 1776-1783, doi:10.1161/circulationaha.107.698662 (2007).

13 Iwatsubo, K. et al. Dopamine induces apoptosis in young, but not in neonatal, neurons via Ca2+-dependent signal. Am. J. Physiol. Cell Physiol. 293, C1498-C1508, doi:10.1152/ajpcell.00088.2007 (2007).

14 Yan, L. et al. Type 5 adenylyl cyclase disruption increases longevity and protects against stress. Cell 130, 247-258, doi:10.1016/j.cell.2007.05.038 (2007).

15 Yagisawa, Y. et al. Effects of occlusal disharmony on cardiac fibrosis, myocyte apoptosis and myocyte oxidative DNA damage in mice. PLoS One 15, e0236547, doi:10.1371/journal.pone.0236547 (2020).

16 Suita, K. et al. Effects of occlusal disharmony on susceptibility to atrial fibrillation in mice. Sci. Rep. 10, 13765, doi:10.1038/s41598-020-70791-8 (2020).

17 Iwatsubo, K. et al. Prevention of heart failure in mice by an antiviral agent that inhibits type 5 cardiac adenylyl cyclase. Am. J. Physiol. Heart Circ. Physiol. 302, H2622-H12628, doi:10.1152/ajpheart.00190.2012 (2012). 
18 Wada, T. et al. Antiviral drug vidarabine possessing cardiac type 5 adenylyl

cyclase inhibitory property did not affect cardiohemodynamic or

electrophysiological variables in the halothane-anesthetized dogs. J. Toxicol. Sci.

41, 115-122, doi:10.2131/jts.41.115 (2016).

19 Shimizu, Y., Khan, M., Kato, G. \& Aoki, K. Occlusal disharmony-induced stress causes osteopenia of the lumbar vertebrae and long bones in mice. Sci. Rep. 8 , 173, doi:10.1038/s41598-017-18037-y (2018).

20 Mori, D., Katayama, T., Miyake, H., Fujiwara, S. \& Kubo, K. Y. Occlusal disharmony leads to learning deficits associated with decreased cellular proliferation in the hippocampal dentate gyrus of SAMP8 mice. Neurosci. Lett. 534, 228-232, doi:10.1016/j.neulet.2012.12.004 (2013).

21 Villalobos, E. et al. Fibroblast primary cilia are required for cardiac fibrosis. Circulation 139, 2342-2357, doi:10.1161/circulationaha.117.028752 (2019).

22 Wilkinson-Berka, J. L., Rana, I., Armani, R. \& Agrotis, A. Reactive oxygen species, Nox and angiotensin II in angiogenesis: implications for retinopathy. Clin. Sci. (Lond) 124, 597-615, doi:10.1042/cs20120212 (2013).

23 Kuroda, J. \& Sadoshima, J. NADPH oxidase and cardiac failure. J. Cardiovasc. Transl. Res. 3, 314-320, doi:10.1007/s12265-010-9184-8 (2010). 
24 Yang, Y. et al. Xanthine oxidase inhibitor allopurinol prevents oxidative stress-mediated atrial remodeling in alloxan-induced diabetes mellitus rabbits. $J$. Am. Heart Assoc. 7, doi:10.1161/jaha.118.008807 (2018).

25 Boueiz, A., Damarla, M. \& Hassoun, P. M. Xanthine oxidoreductase in respiratory and cardiovascular disorders. Am. J. Physiol. Lung Cell Mol. Physiol. 294, L830-L840, doi:10.1152/ajplung.00007.2008 (2008).

26 Zhao, H. et al. Cinnamaldehyde ameliorates LPS-induced cardiac dysfunction via TLR4-NOX4 pathway: The regulation of autophagy and ROS production. $J$. Mol. Cell Cardiol. 101, 11-24, doi:10.1016/j.yjmcc.2016.10.017 (2016).

27 Bode, J. G., Ehlting, C. \& Häussinger, D. The macrophage response towards LPS and its control through the p38(MAPK)-STAT3 axis. Cell Signal. 24, 1185-1194, doi:10.1016/j.cellsig.2012.01.018 (2012).

28 Matesanz, N. et al. Xanthine oxidase-derived extracellular superoxide anions stimulate activator protein 1 activity and hypertrophy in human vascular smooth muscle via c-Jun $\mathrm{N}$-terminal kinase and p38 mitogen-activated protein kinases. $J$. Hypertens. 25, 609-618, doi:10.1097/HJH.0b013e328013e7c4 (2007).

29 Zhang, T., Zhang, Y. \& Cui, M. CaMKII is a RIP3 substrate mediating ischemiaand oxidative stress-induced myocardial necroptosis. Nat. Med. 22, 175-182, 
doi:10.1038/nm.4017 (2016).

30 Chen, W. J. et al. Tachycardia-induced CD44/NOX4 signaling is involved in the development of atrial remodeling. J. Mol .Cell Cardiol. 135, 67-78, doi:10.1016/j.yjmcc.2019.08.006 (2019).

31 Cheng, H. \& Lederer, W. J. Calcium sparks. Physiol. Rev. 88, 1491-1545, doi:10.1152/physrev.00030.2007 (2008).

32 Mattila, K. J. et al. Association between dental health and acute myocardial infarction. B.M.J. 298, 779-781, doi:10.1136/bmj.298.6676.779 (1989).

33 Joshipura, K. J., Douglass, C. W. \& Willett, W. C. Possible explanations for the tooth loss and cardiovascular disease relationship. Ann. Periodontol. 3, 175-183, doi:10.1902/annals.1998.3.1.175 (1998).

34 Wu, D. \& Liu, J. Occlusal interference induces oxidative stress and increases the expression of UCP3 in the masseter muscle: A rat model. Arch. Oral Biol. 102, 249-255, doi:10.1016/j.archoralbio.2019.04.022 (2019).

35 Iyomasa, M. M. et al. Histological and histochemical effects after occlusion alteration in suprahyoid muscles. Micron 40, 239-246, doi:10.1016/j.micron.2008.08.001 (2009).

36 Buczko, P., Zalewska, A. \& Szarmach, I. Saliva and oxidative stress in oral 
cavity and in some systemic disorders. J. Physiol. Pharmacol. 66, 3-9 (2015).

37 Vatner, S. F. et al. Adenylyl cyclase type 5 in cardiac disease, metabolism, and aging. Am. J. Physiol. Heart Circ. Physiol. 305, H1-H8, doi:10.1152/ajpheart.00080.2013 (2013).

38 Vatner, S. F., Pachon, R. E. \& Vatner, D. E. Inhibition of adenylyl cyclase type 5 increases longevity and healthful aging through oxidative stress protection. Oxid. Med. Cell Longev. 2015, 250310, doi:10.1155/2015/250310 (2015).

39 Ishikawa, Y. \& Homcy, C. J. The adenylyl cyclases as integrators of transmembrane signal transduction. Circ. Res. 80, 297-304, doi:10.1161/01.res.80.3.297 (1997).

40 Espinasse, I. et al. Type V, but not type VI, adenylyl cyclase mRNA accumulates in the rat heart during ontogenic development. Correlation with increased global adenylyl cyclase activity. J. Mol. Cell. Cardiol. 27, 1789-1795, doi:10.1016/0022-2828(95)90002-0 (1995).

41 Okumura, S. et al. Disruption of type 5 adenylyl cyclase gene preserves cardiac function against pressure overload. Proc. Natl. Acad. Sci. USA 100, 9986-9990, doi:10.1073/pnas.1733772100 (2003).

42 Hung, H. C. et al. The association between tooth loss and coronary heart disease 
in men and women. J. Public Health Dent. 64, 209-215,

doi:10.1111/j.1752-7325.2004.tb02755.x. (2004).

43 Ikebe, K. et al. Masticatory performance in older subjects with varying degrees of tooth loss. J. Dent. 40, 71-76, doi:10.1016/j.jdent.2011.10.007 (2012).

44 Ikebe, K. et al. Association of masticatory performance with age, gender, number of teeth, occlusal force and salivary flow in Japanese older adults: is ageing a risk factor for masticatory dysfunction? Arch. Oral Biol. 56, 991-996, doi:10.1016/j.archoralbio.2011.03.019 (2011).

45 Kugimiya, Y. et al. Factors associated with masticatory performance in community-dwelling older adults: A cross-sectional study. J. Am. Dent. Assoc.151, 118-126, doi:10.1016/j.adaj.2019.10.003 (2020).

46 Iwashima, Y. et al. Additive interaction of oral health disorders on risk of hypertension in a Japanese urban population: the Suita Study. Am. J. Hypertens. 27, 710-719, doi:10.1093/ajh/hpt227 (2014).

47 Katayama, T. et al. Effect of bite-raised condition on the hippocampal cholinergic system of aged SAMP8 mice. Neurosci. Lett. 520, 77-81, doi:10.1016/j.neulet.2012.05.035 (2012).

48 Suita, K. et al. Vidarabine, an anti-herpesvirus agent, prevents 
catecholamine-induced arrhythmias without adverse effect on heart function in mice. Pflugers Arch. 470, 923-935, doi:10.1007/s00424-018-2121-4 (2018).

49 Suita, K. et al. Norepinephrine-Induced Adrenergic Activation Strikingly Increased the Atrial Fibrillation Duration through $\beta_{1}$ - and $\alpha_{1}$-adrenergic receptor-mediated signaling in mice. PLoS One 10, e0133664, doi:10.1371/journal.pone.0133664 (2015).

50 Kawamura, N. et al. Effects of chronic Porphyromonas gingivalis lipopolysaccharide infusion on skeletal muscles in mice. J. Physiol. Sci. 69, 503-511, doi:10.1007/s12576-019-00670-z (2019).

51 Ito, A. et al. Role of $\beta$-adrenergic signaling in masseter muscle. PLoS One 14, e0215539, doi:10.1371/journal.pone.0215539 (2019).

52 Bai, Y. et al. Pharmacological stimulation of type 5 adenylyl cyclase stabilizes heart rate under both microgravity and hypergravity induced by parabolic flight. J. Pharmacol. Sci. 119, 381-389, doi:10.1254/jphs.12102fp (2012).

53 Umeki, D. et al. Protective Effects of Clenbuterol against Dexamethasone-induced masseter muscle atrophy and myosin heavy chain transition. PLoS One 10, e0128263, doi:10.1371/journal.pone.0128263 (2015).

54 Kilkenny, C. et al. Survey of the quality of experimental design, statistical 
analysis and reporting of research using animals. PLoS One 4, e7824,

doi:10.1371/journal.pone.0007824 (2009).

55 National Research Council (US) Committee for the Update of the Guide for the Care and Use of Laboratory Animals. Guide for the Care and Use of Laboratory Animals. 8th edition. Washington (DC): National Academies Press (US) National Academy of Sciences, doi:10.17226/12910 (2011).

56 Okumura, S. et al. Epacl-dependent phospholamban phosphorylation mediates the cardiac response to stresses. J. Clin. Invest. 124, 2785-2801, doi:10.1172/jci64784 (2014).

57 Yan, J. et al. Novel methods of automated quantification of gap junction distribution and interstitial collagen quantity from animal and human atrial tissue sections. PLoS One 9, e104357, doi:10.1371/journal.pone.0104357 (2014).

58 Stromp, T. A. et al. Quantitative gadolinium-free cardiac fibrosis imaging in end stage renal disease patients reveals a longitudinal correlation with structural and functional decline. Sci. Rep. 8, 16972, doi:10.1038/s41598-018-35394-4 (2018).

59 Yu, H. et al. The rat IgGFc $\gamma$ BP and Muc2 C-terminal domains and TFF3 in two intestinal mucus layers bind together by covalent interaction. PLoS One 6, 
e20334, doi:10.1371/journal.pone.0020334 (2011). 


\section{Figure legends}

\section{Figure 1.}

Schematic illustrations of experimental procedure and bite-opening treatment, and comparison of body weight, cardiac muscle weight and lung weight among the groups.

(A) Male 16-week-old C57BL/6 mice were divided into four groups: a normal control group (CTRL), a bite-opening (BO)-treated group, a vidarabine-treated group (V), and a $\mathrm{BO}$ plus vidarabine-treated $(\mathrm{BO}+\mathrm{V})$ group. Long-term infusion of vidarabine was performed for 14 days at a dose of $15 \mathrm{mg} / \mathrm{kg} /$ day with the osmotic mini-pumps, and the indicated measurements were made

(B) Schematic representation of a bite-opening (BO) in the form of a $0.7 \mathrm{~mm}$ increase in the vertical height of occlusion, obtained by cementing a composite resin onto the mandibular incisors to cause occlusal disharmony in mice.

(C) Body weight was measured daily for all animals throughout the 2-week experimental period. ${ }^{* * * *} P<0.0001$ (Control $(n=5)$ vs. $\mathrm{BO}(n=5),{ }^{\# \# \#} P<0.0001$ (Control vs. $\mathrm{BO}+\mathrm{V}(n=5),{ }^{+1+} P<0.0001$ (BO vs. $\left.\mathrm{V}(n=5)\right),{ }^{*} * * * 3<0.0001$ (V vs. $\mathrm{BO}+\mathrm{V}$ ) by two-way repeated-measures ANOVA followed by the Bonferroni post hoc test. 
(D, E) No significant difference in heart (C) or lung (D) size in terms of weight per tibial length ratio $(\mathrm{mg} / \mathrm{mm})$ at 2 weeks after $\mathrm{BO}$ treatment (Control $(n=5), \mathrm{BO}(n=5)$, vidarabine $(n=5)$ and $\mathrm{BO}+$ vidarabine $(n=5)$ groups; $P=\mathrm{NS}$, not significantly different, vs. Control by one-way ANOVA) followed by the Tukey-Kramer post hoc test. Data are presented as mean $\pm \mathrm{SD}$.

\section{Figure 2.}

\section{Effect of vidarabine on BO-induced fibrosis in the heart.}

(A) Representative images of Masson-trichrome-stained sections of cardiac muscle in the Control (CTRL) (upper left), BO (upper right), vidarabine (V) (lower left) and $\mathrm{BO}+$ $\mathrm{V}$; lower right) groups.

(B) The area of fibrosis was significantly increased in the BO group $(n=5)$, but this increase was blocked in the $\mathrm{BO}+\mathrm{V}$ group $(n=4) .{ }^{* *} P<0.01,{ }^{* * *} P<0.001$ by one-way repeated-measures ANOVA followed by the Tukey-Kramer post hoc test.

(C) Expression of $\alpha$-SMA, a fibrosis-related gene, was significantly increased in the BO group $(n=4)$, but this increase was blocked in the $\mathrm{BO}+\mathrm{V}$ group $(n=4) .{ }^{*} P<0.05,{ }^{* *} P$ $<0.01$ by one-way ANOVA followed by the Tukey-Kramer post hoc test. Data are 
presented as mean \pm SD. Full-size images of immunoblots are presented in

\section{Supplementary Fig. 2.}

\section{Figure 3.}

\section{Effect of vidarabine on BO-induced cardiac myocyte apoptosis.}

(A) TUNEL-positive nuclei (black arrows) in representative TUNEL-stained sections were counted in cardiac muscle in the Control (CTRL; upper left), BO (upper right),

Vidarabine $(\mathrm{V}$; lower left $)$ and $\mathrm{BO}+\mathrm{V}($ lower right $)$ groups.

(B) The number of TUNEL-positive nuclei was significantly increased in the BO group $(n=5)$, but this increase was blocked in the $\mathrm{BO}+\mathrm{V}$ group $(n=4) . \stackrel{*}{P}<0.05$ by one-way ANOVA followed by the Tukey-Kramer post hoc test.

(C) The Bax/BCL-2 ratio was significantly increased in the BO group $(n=4)$, but this increase was blocked in the $\mathrm{BO}+\mathrm{V}$ group $(n=5) . \stackrel{*}{P}<0.05$ by one-way ANOVA followed by the Tukey-Kramer post hoc test. Data are presented as mean \pm SD. Full-size images of immunoblots are presented in Supplementary Fig. 3.

\section{Figure 4.}

Effect of vidarabine on BO-induced oxidative stress in cardiac muscle. 
(A) Representative immunohistochemical images of oxidative DNA damage (8-OHdG) in cardiac muscle in the Control (CTRL; upper left), BO (upper right), vidarabine (V; lower left) and $\mathrm{BO}+\mathrm{V}$ (lower right) groups.

(B) 8-OHdG-positive nuclei were significantly increased in the BO group $(n=5)$, but this increase was blocked in the BO $+\mathrm{V}$ group $(n=4) .{ }^{*} P<0.05$ by one-way ANOVA followed by the Tukey-Kramer post hoc test.

(C) Representative SDS-PAGE of oxidized proteins in cardiac muscle homogenate prepared from Control (CTRL; lane 1), BO (lane 2), V (lane 3) and BO + V (lane 4) groups using the OxiSelect ${ }^{\mathrm{TM}}$ Protein Carbonyl Immunoblot Kit. Full-size images of immunoblots are presented in Supplementary Fig. 5.

(D) Oxidized proteins were significantly increased in the BO group $(n=5)$, but this increase was blocked in the $\mathrm{BO}+\mathrm{V}$ group $(n=4) .{ }^{*} P<0.05,{ }^{* *} P<0.01$ by one-way ANOVA followed by the Tukey-Kramer post hoc test.

Data are presented as mean \pm SD.

\section{Figure 5.}

Effect of vidarabine on NOX4/2, XO and phospho-p38 in the heart of BO mice. 
(A) NOX4 expression was significantly increased in the BO group $(n=4)$, and this increase was significantly blocked in the $\mathrm{BO}+\mathrm{V}$ group $(n=5) .{ }^{*} P<0.05,{ }^{* *} P<0.01$ by one-way ANOVA followed by the Tukey-Kramer post hoc test. Full-size images of immunoblots are presented in Supplementary Fig. 6.

(B) NOX2 expression was similar among the four groups $(n=5$ each). NS, not significantly different, by one-way ANOVA followed by the Tukey-Kramer post hoc test. Full-size images of immunoblots are presented in Supplementary Fig. 7.

(C) Expression of XO was significantly increased in the BO group $(n=4)$, and this increase was significantly blocked in the $\mathrm{BO}+\mathrm{V}$ group $(n=4) .{ }^{*} P<0.05, \quad{ }^{* *} P<0.01$, ${ }^{* * *} P<0.001$ by one-way ANOVA followed by the Tukey-Kramer post hoc test.

Full-size images of immunoblots are presented in Supplementary Fig. 8.

(D) Expression of phospho-p38 was significantly increased in the BO group $(n=4)$ and this was significantly blocked in the $\mathrm{BO}+\mathrm{V}$ group $(n=5) .{ }^{*} P<0.05$ by one-way ANOVA followed by the Tukey-Kramer post hoc test. Full-size images of immunoblots are presented in Supplementary Fig. 9. Data are presented as mean \pm SD.

\section{Figure 6.}

Effect of vidarabine on BO-induced RIP3, phospho-CaMKII and phospho-PLN in 


\section{the heart.}

(A) Expression of RIP3, a key mediator of necroptosis, was significantly increased in the BO group $(n=4)$, but this increase was significantly blocked in the $\mathrm{BO}+\mathrm{V}$ group $(n=4) .{ }^{* *} P<0.01, \quad{ }^{* * *} P<0.001$ by one-way ANOVA followed by the Tukey-Kramer post hoc test. Full-size images of immunoblots are presented in Supplementary Fig.

10.

(B) Expression of phospho-CaMKII (Thr-286) was significantly increased in the BO group $(n=5)$, but this increase was significantly blocked in the BO $+\mathrm{V}$ group $(n=4)$. ${ }^{*} P<0.05, \quad{ }^{* *} P<0.01, \quad{ }^{* * *} P<0.001$ by one-way ANOVA followed by the Tukey-Kramer post hoc test. Full-size images of immunoblots are presented in

\section{Supplementary Fig. 11.}

(C) Expression of phospho-PLN (Thr-17) was significantly increased in the BO group $(n=5)$, but this increase was blocked in the $\mathrm{BO}+\mathrm{V}$ group $(n=4) . \stackrel{*}{P}<0.05, \stackrel{* *}{P} P<$ 0.01 by one-way ANOVA followed by the Tukey-Kramer post hoc test. Full-size images of immunoblots are presented in Supplementary Fig. 12.

(D) Expression of phospho-PLN (Ser-16) was significantly increased in the BO group $(n=5)$, but this increase was significantly blocked in the BO $+\mathrm{V}$ group $(n=5) . \stackrel{*}{P}<$ $0.05, \quad,^{* *} P<0.01$ by one-way ANOVA followed by the Tukey-Kramer post hoc test. 
Full-size images of immunoblots are presented in Supplementary Fig. 13. Data are presented as expressed as mean $\pm \mathrm{SD}$.

\section{Figure 7.}

This scheme illustrates the proposed role of $\beta-\mathrm{AR} / \mathrm{Gs} \alpha / \mathrm{AC} 5$ signaling in the heart of $\mathrm{BO}$ mice. $\beta-\mathrm{AR} / \mathrm{Gs} \alpha / \mathrm{AC} 5$ signaling is activated by the $\mathrm{BO}$ treatment, leading to oxidative stress and phosphorylation of CaMKII (Thr-286), which mediates PLN phosphorylation at Thr-17. In addition, cAMP derived from AC5 mediates oxidative stress and PLN phosphorylation at Ser-16. These changes might cause fibrosis, myocyte apoptosis and oxidative stress in the heart of BO mice, leading to cardiac dysfunction. 
Fig.1
A
B

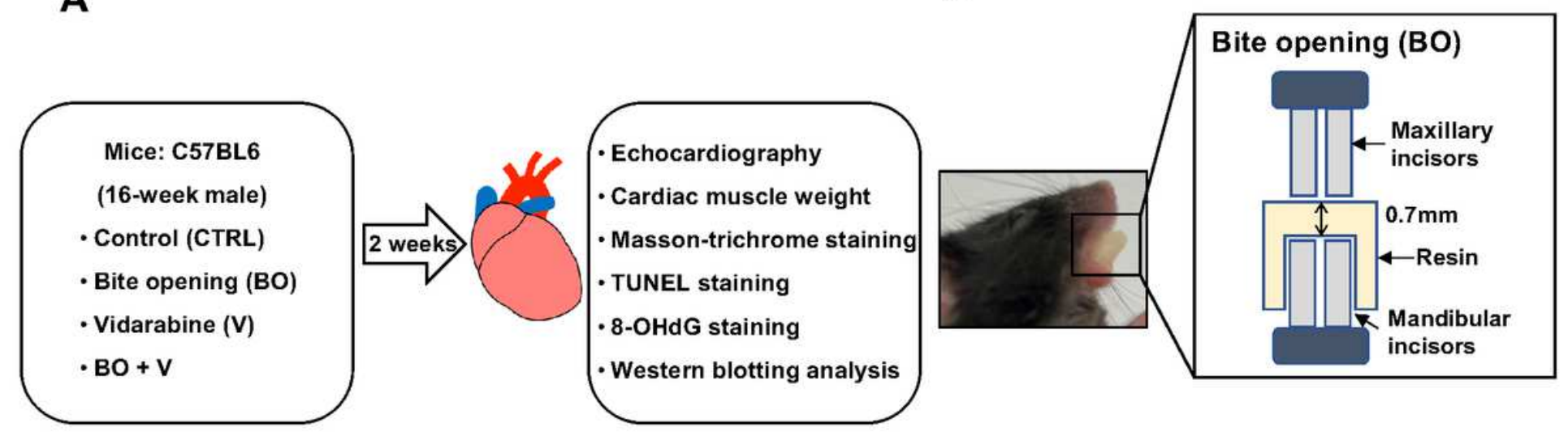

C

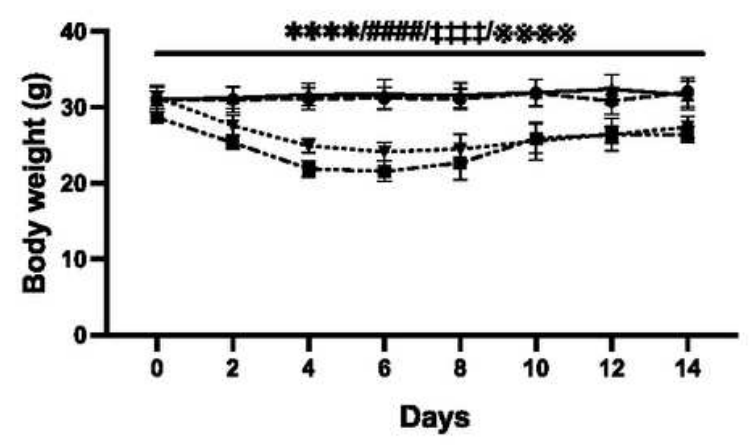

D

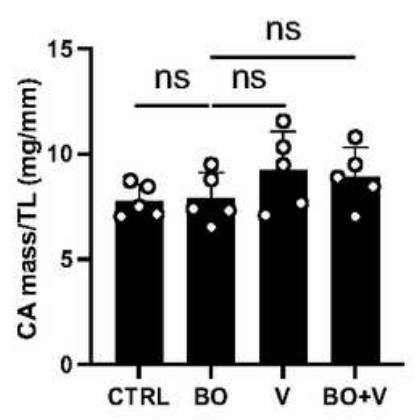

$\mathbf{E}$

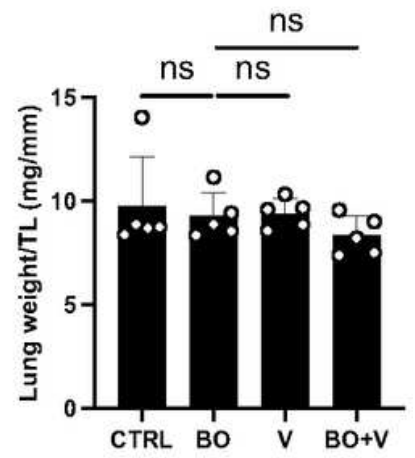

\section{Figure 1}

Schematic illustrationsof experimental procedureand bite-opening treatment, and comparison of body weight, cardiac muscle weight and lung weightamong the groups. (A)Male 16-week-old C57BL/6 mice were divided into four groups:a normal control group (CTRL), a bite-opening (BO)-treated group, a vidarabine-treated group $(\mathrm{V})$, and a $\mathrm{BO}$ plus vidarabine-treated $(\mathrm{BO}+\mathrm{V})$ group. Long-term infusion of vidarabine was performed for 14 days at a dose of $15 \mathrm{mg} / \mathrm{kg} /$ day with the osmotic mini-pumps, and the indicated measurements were made (B)Schematic representation of a bite-opening (BO) in the form of a $0.7 \mathrm{~mm}$ increase in the vertical height of occlusion, obtained by cementing a composite resin onto the mandibular incisors to cause occlusal disharmony in mice. (C) Body weight was measured daily for all animals throughout the 2-week experimental period. ${ }^{\star * \star *} P<0.0001$ (Control $(n=5)$ vs. $B O(n=5)$,\#\#\#\#P<

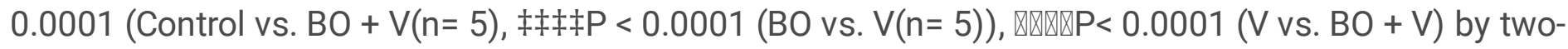
way repeated-measures ANOVA followed by the Bonferroni post hoctest.39 (D, E) No significant difference in heart (C)orlung (D)size in terms of weight per tibial length ratio $(\mathrm{mg} / \mathrm{mm})$ at 2 weeks after 
BO treatment(Control $(n=5), B O(n=5)$, vidarabine $(n=5)$ and BO + vidarabine $(n=5)$ groups; $P=N S$, not significantly different, vs.Control by one-way ANOVA)followed by the Tukey-Kramer post hoc test.Data are presented as mean \pm SD.

Fig. 2

A
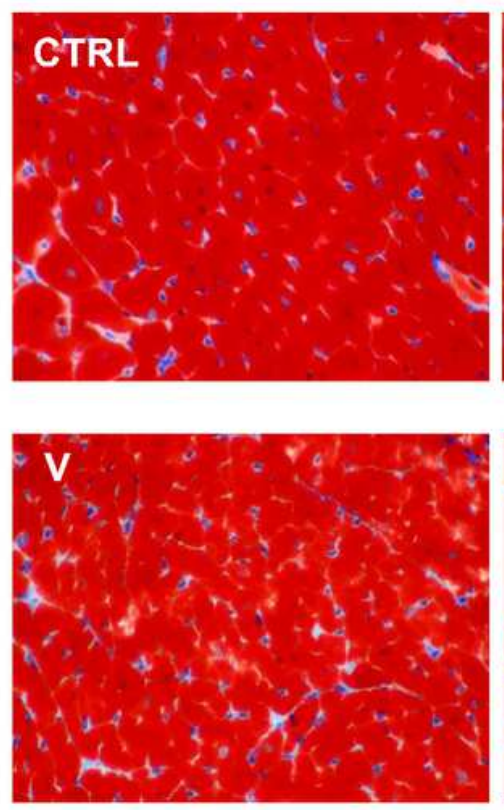
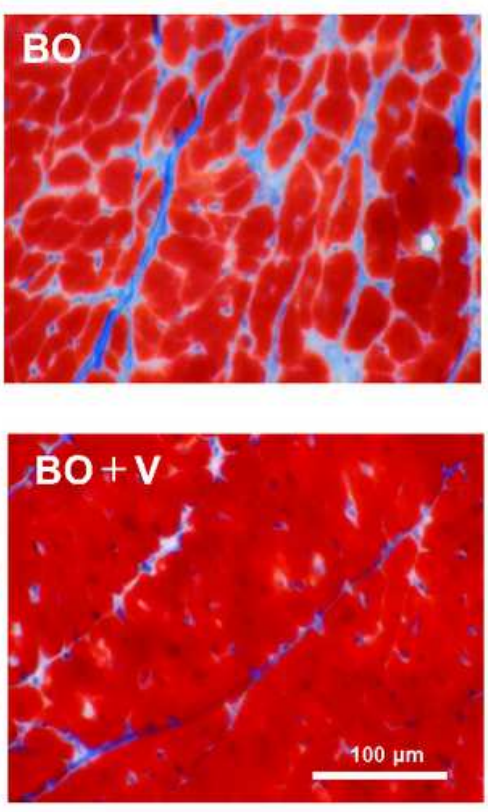

B

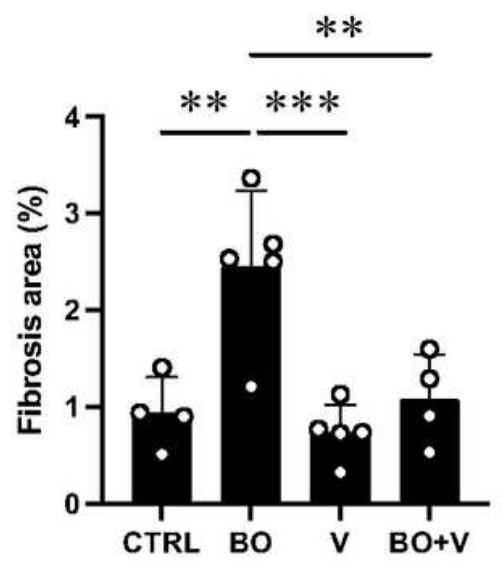

C

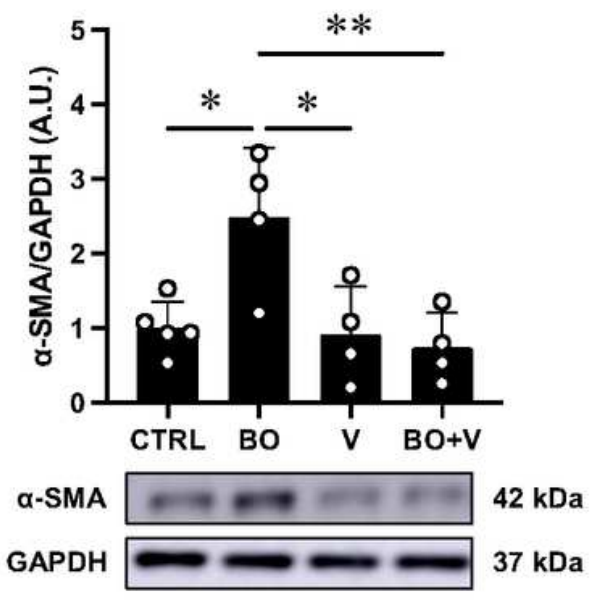

\section{Figure 2}

Effect of vidarabine on BO-induced fibrosis in the heart. (A) Representative images of Masson-trichromestained sections of cardiac muscle in the Control (CTRL) (upper left), BO (upper right), vidarabine (V) (lower left) and BO + V; lower right) groups. (B) The area of fibrosis was significantly increased in the BO group $(n=5)$, but this increase was blocked in the BO $+V$ group $(n=4) . \star \star P<0.01, \star \star \star P<0.001$ by oneway repeated-measures ANOVA followed by the Tukey-Kramer post hoc test. (C) Expression of a-SMA, a fibrosis-related gene, was significantly increased in the BO group $(n=4)$, but this increase was blocked in the BO $+V$ group $(n=4) .{ }^{*} P<0.05,{ }^{* *} P<0.01$ by one-way ANOVA followed by the Tukey-Kramer post hoc test. Data are 40 presented as mean \pm SD. Full-size images of immunoblots are presented in Supplementary Fig. 2. 
A
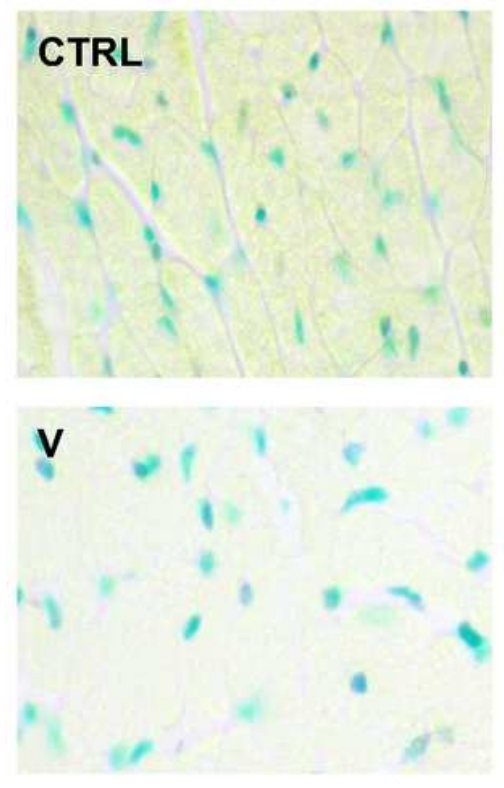
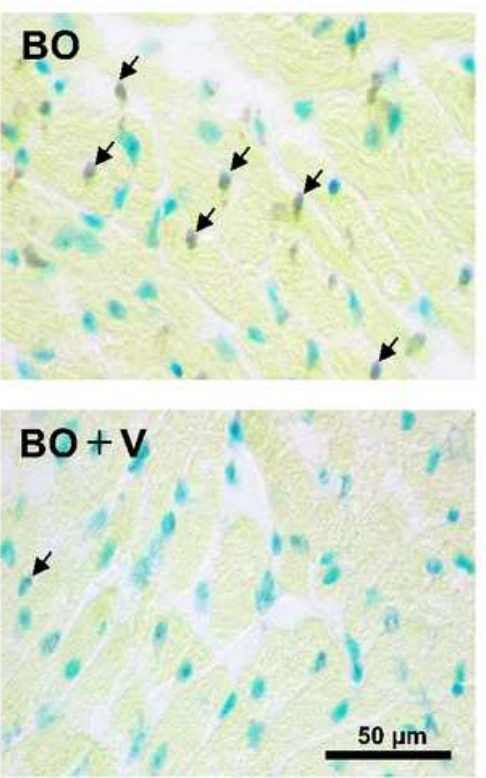

B

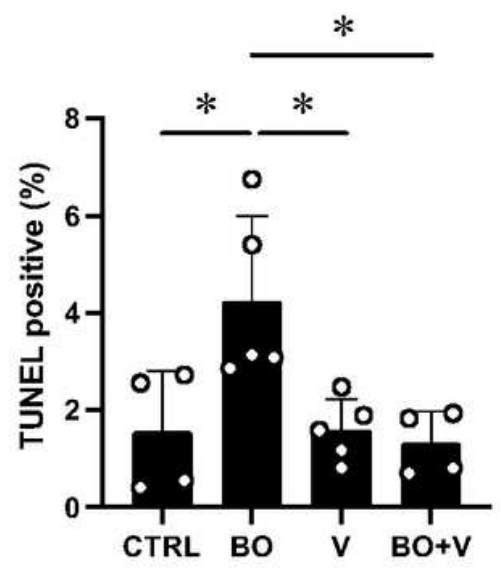

C

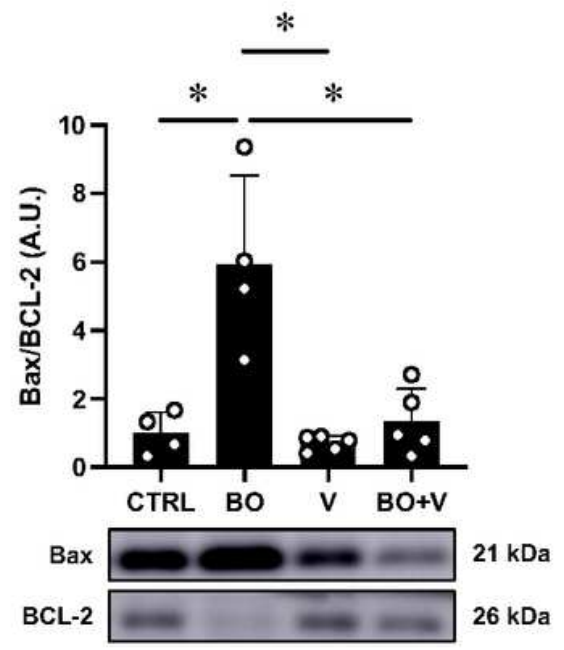

\section{Figure 3}

Effect of vidarabine on BO-induced cardiac myocyte apoptosis. (A) TUNEL-positive nuclei (black arrows) in representative TUNEL-stained sections were counted in cardiac muscle in the Control (CTRL; upper left), BO (upper right), Vidarabine ( $\mathrm{V}$; lower left) and BO + V (lower right) groups. (B) The number of TUNEL-positive nuclei was significantly increased in the BO group $(n=5)$, but this increase was blocked in the $B O+V$ group $(n=4) .{ }^{*} P<0.05$ by one-way ANOVA followed by the Tukey-Kramer post hoc test.

(C)TheBax/BCL-2 ratio was significantly increased in the BO group $(n=4)$, but this increase was blocked in the $B O+V$ group $(n=5)$. ${ }^{*}<0.05$ by one-way ANOVA followed by the Tukey-Kramer post hoc test. Data are presented as mean \pm SD. Full-size images of immunoblots are presented in Supplementary Fig. 3. 
A

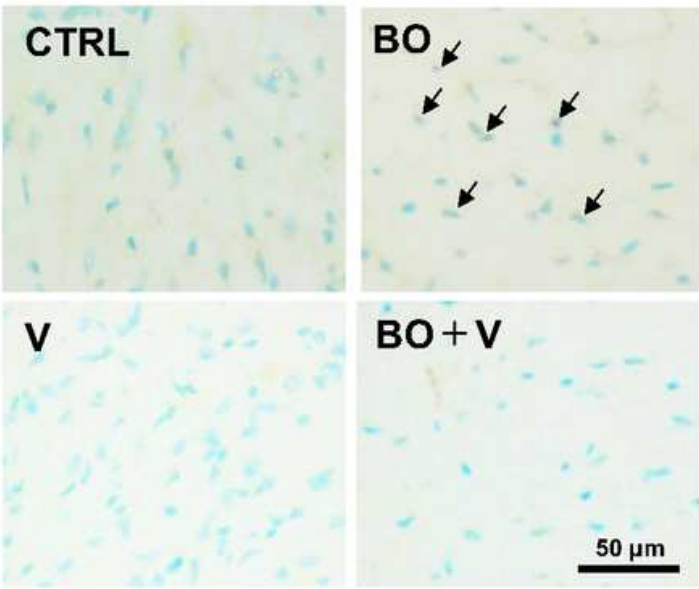

C

\section{Oxidized protein}

GAPDH
B

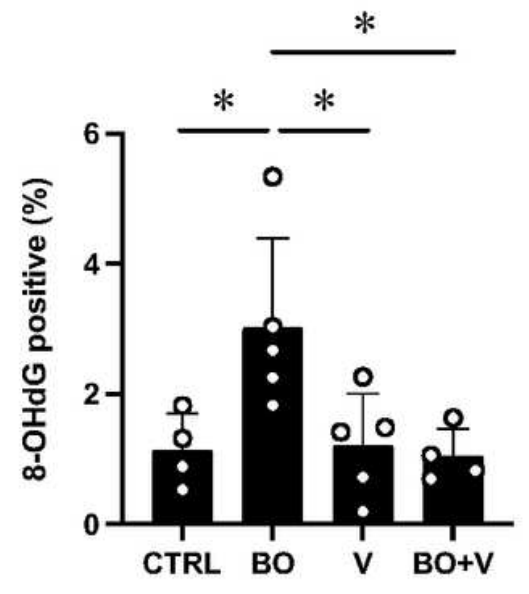

D

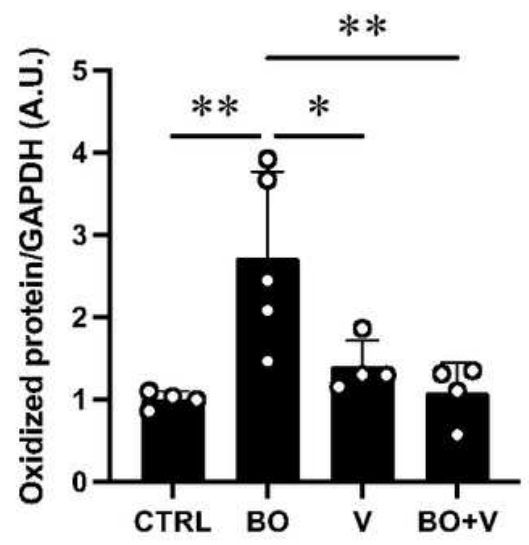

\section{Figure 4}

Effect of vidarabine on BO-induced oxidative stress in cardiac muscle. 41 (A) Representative immunohistochemical images of oxidative DNA damage (8-OHdG) in cardiac muscle in the Control (CTRL; upper left), BO (upper right), vidarabine (V; lower left) and BO + V (lower right) groups. (B) 8-OHdGpositive nuclei were significantly increased in the BO group $(n=5)$, but this increase was blocked in the $\mathrm{BO}+\mathrm{V}$ group $(\mathrm{n}=4) .{ }^{*} \mathrm{P}<0.05$ by one-way ANOVA followed by the Tukey-Kramer post hoc test. (C) Representative SDS-PAGE of oxidized proteins in cardiac muscle homogenate prepared from Control (CTRL; lane 1), BO (lane 2), V (lane 3) and BO + V (lane 4) groups using the OxiSelectTMProtein Carbonyl Immunoblot Kit. Full-size images of immunoblots are presented in Supplementary Fig. 5. (D) Oxidized proteins were significantly increased in the BO group $(n=5)$, but this increase was blocked in the BO $+V$ group $(n=4) .{ }^{*} P<0.05,{ }^{*} P<<0.01$ by one-way ANOVA followed by the Tukey-Kramer post hoc test. Data are presented as mean \pm SD. 
Fig.5 A

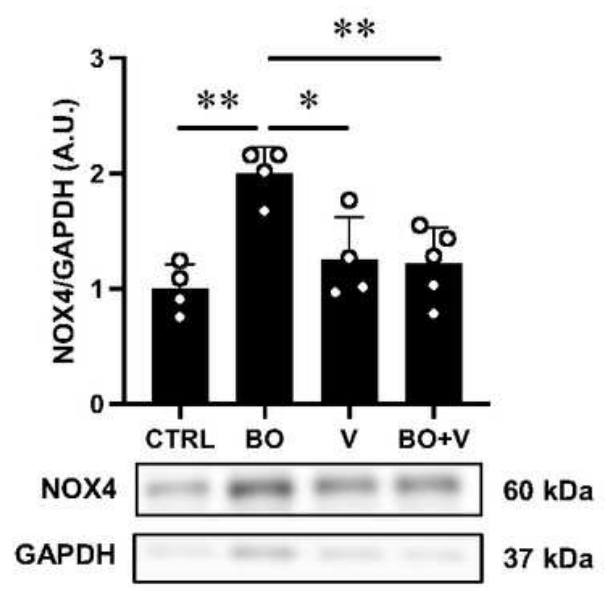

C

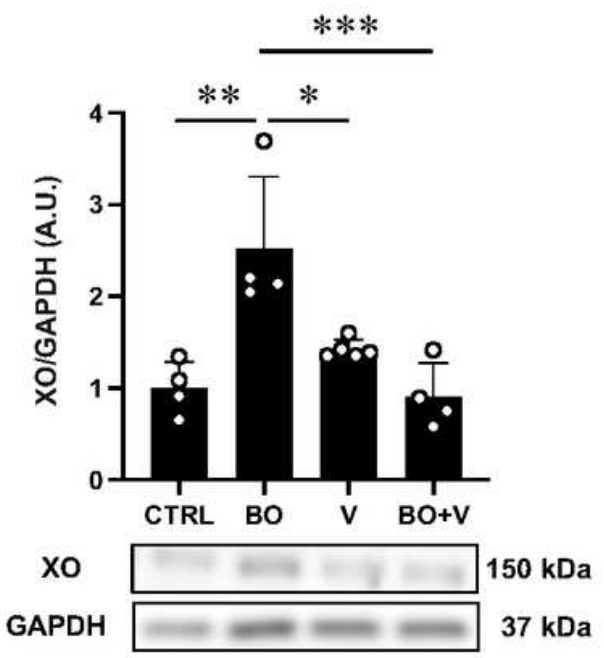

B

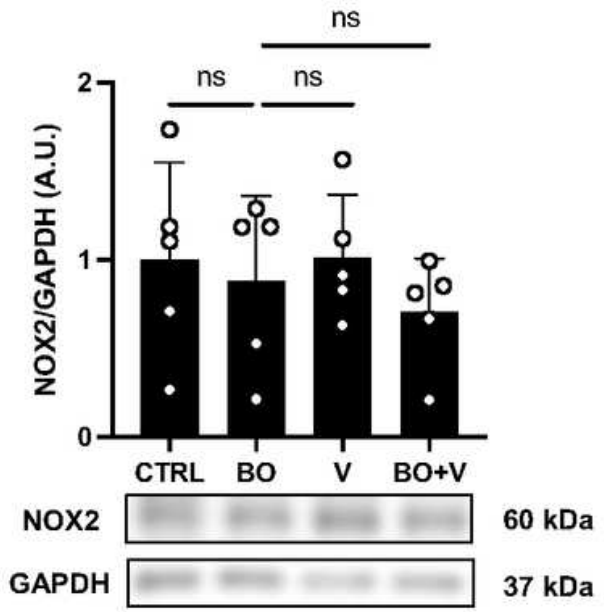

D

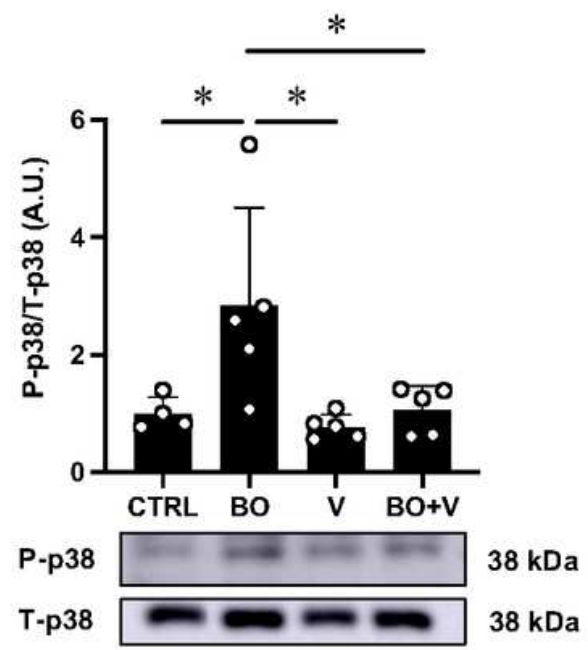

\section{Figure 5}

Effect of vidarabine on NOX4/2, XO and phospho-p38 in the heart of BO mice. 42 (A) NOX4 expression was significantly increased in the BO group $(n=4)$, and this increase was significantly blocked in the BO $+V$ group $(n=5) .{ }^{*} P<0.05,{ }^{*} P<0.01$ by one-way ANOVA followed by the Tukey-Kramer post hoc test. Full-size images of immunoblots are presented in Supplementary Fig. 6. (B) NOX2 expression was similar among the four groups ( $\mathrm{n}=5$ each). NS, not significantly different, by one-way ANOVA followed by the Tukey-Kramer post hoc test. Full-size images of immunoblots are presented in Supplementary Fig. 7. (C) Expression of $X O$ was significantly increased in the BO group $(n=4)$, and this increase was significantly blocked in the $B O+V$ group $(n=4) .{ }^{*}<<0.05,{ }^{*} P<0.01,{ }^{*} * \mathrm{P}<0.001$ by one-way ANOVA followed by the Tukey-Kramer post hoc test. Full-size images of immunoblots are presented in Supplementary Fig. 8. (D) Expression of phospho-p38was significantly increased in the BO group $(n=4)$ and this was significantly blocked in the BO $+V$ group $(n=5)$. ${ }^{*}<0.05$ by one-way ANOVA followed by the Tukey-Kramer post hoc test. Full-size images of immunoblots are presented in Supplementary Fig. 9. Data are presented as mean \pm SD. 
Fig. 6

A

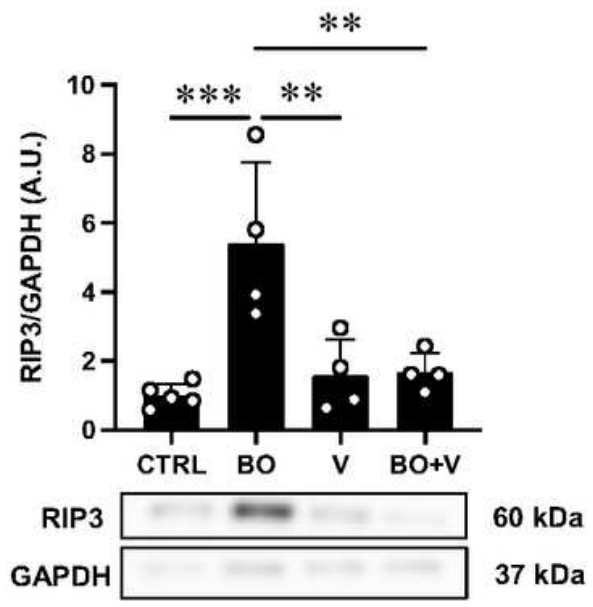

C

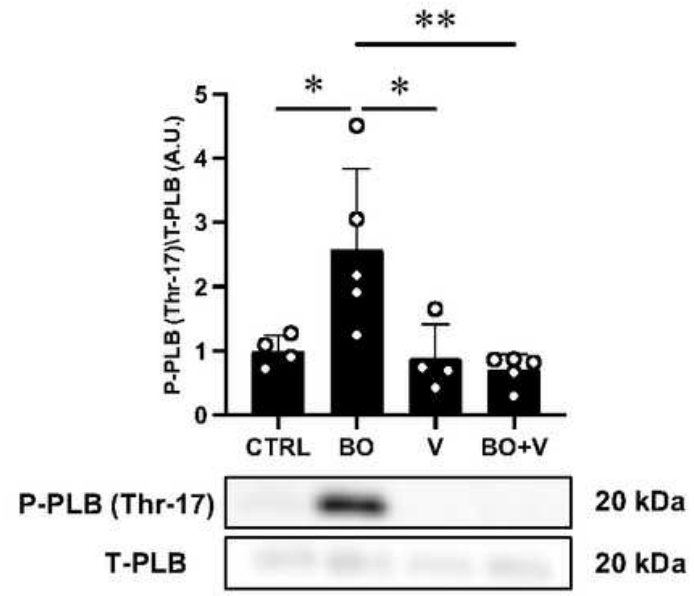

B

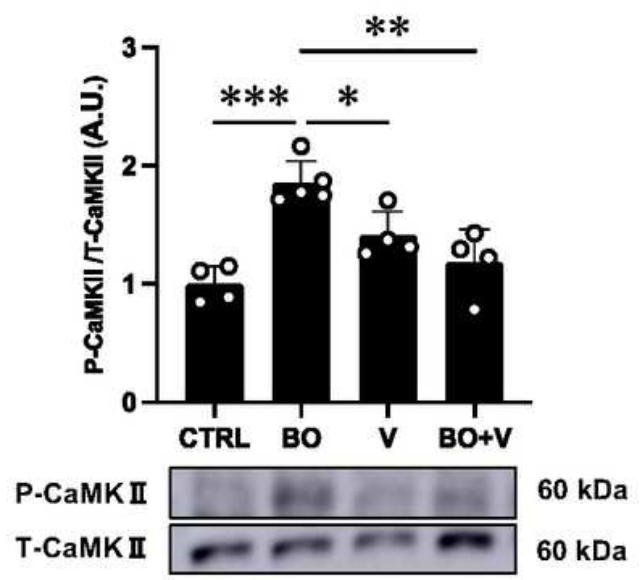

D

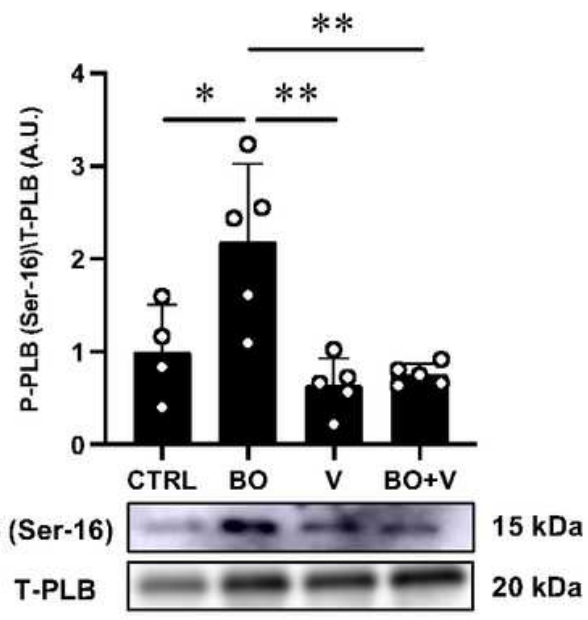

Figure 6

Effectof vidarabine on BO-induced RIP3, phospho-CaMKII and phospho-PLNin 43 the heart. (A) Expression of RIP3, a key mediator of necroptosis, was significantly increased in the BO group $(n=4)$, but this increase was significantly blocked in the BO $+V$ group $(n=4)$. ${ }^{\star \star} P<0.01$, ${ }^{\star \star \star} P<0.001$ by one-way ANOVA followed by the Tukey-Kramer post hoc test. Full-size images of immunoblots are presented in Supplementary Fig. 10. (B) Expression of phospho-CaMKII (Thr-286) was significantly increased in the BO group $(n=5)$, but this increase was significantly blocked in the $B O+V$ group $(n=4) . * P<0.05, * * P<0.01$, $\star \star \star P<0.001$ by one-way ANOVA followed by the Tukey-Kramer post hoc test. Full-size images of immunoblots are presented in 
Figure 7

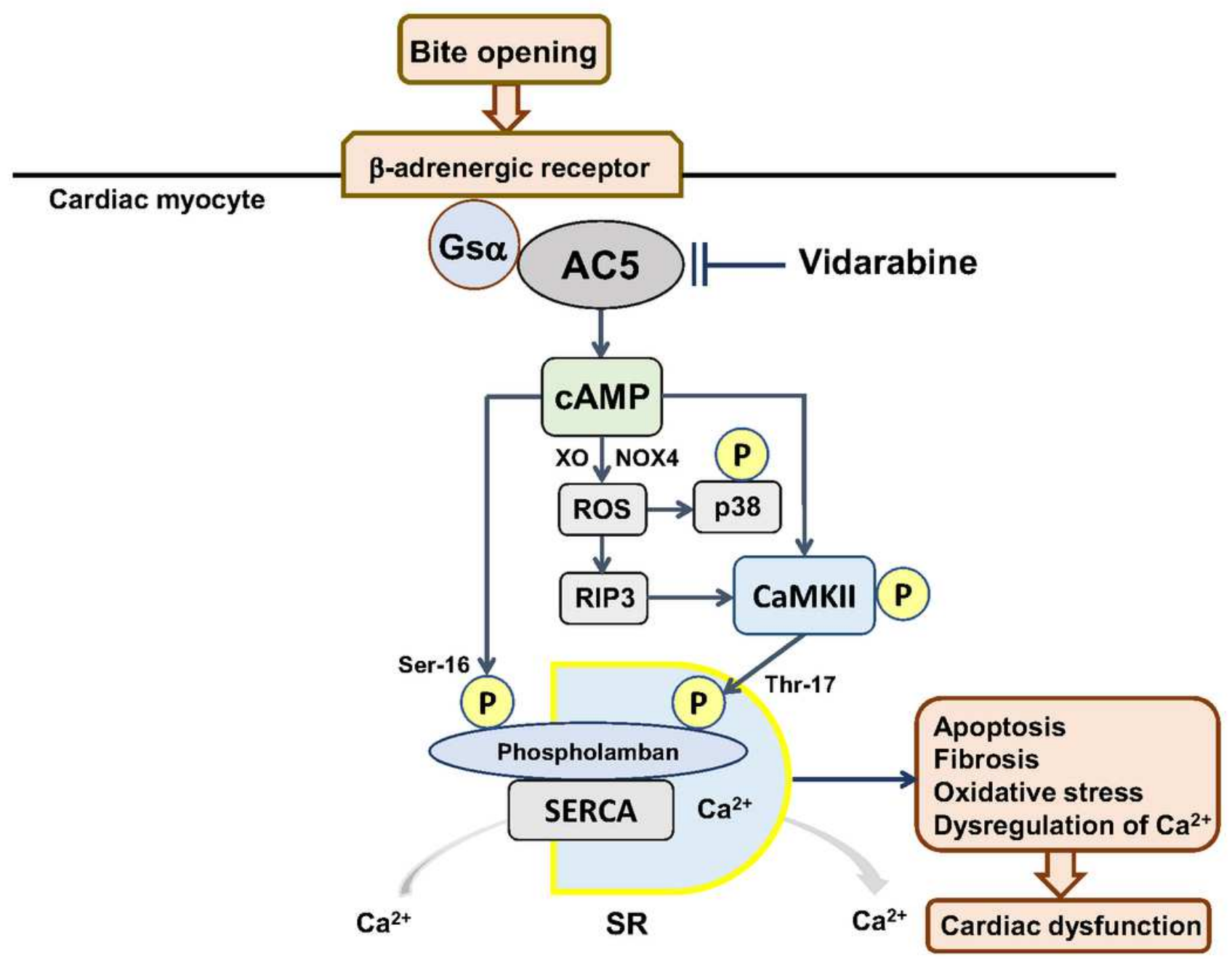

Figure 7

This scheme illustrates the proposed role of $\beta-A R / G s a / A C 5$ signaling in the heart of BO mice. $\beta$ $A R / G s a / A C 5$ signaling is activated by the BO treatment, leading to oxidative stress and phosphorylation of CaMKII (Thr-286), which mediates PLN phosphorylation at Thr-17. In addition, cAMP derived from AC5 mediates oxidative stress and PLN phosphorylation at Ser-16. These changes might cause fibrosis, myocyte apoptosis and oxidative stress in the heart of BO mice, leading to cardiac dysfunction.

\section{Supplementary Files}

This is a list of supplementary files associated with this preprint. Click to download.

- Table1Hayakawaetal..pdf

- SupplementaryDataSci742021.pdf 